\title{
Experimental trends of reflectometry frequency spectra emerging from a systematic analysis of the Tore Supra database
}

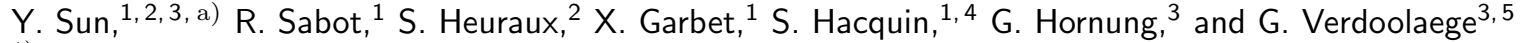 \\ 1) CEA, IRFM, F-13108 Saint Paul Lez Durance, France \\ 2) IJL UMR 7198 CNRS, Université de Lorraine, BP50840, F-54011 Nancy Cedex, \\ France \\ 3) Department of Applied Physics, Ghent University, 9000 Gent, Belgium \\ ${ }^{4)}$ EUROfusion Programme Management Unit, Culham Science Centre, Culham, OX14 3DB, \\ $U K$ \\ 5) LPP-ERM/KMS, B-1000 Brussels, Belgium
}

One major goal of data science applications is to extract patterns from large data sets. Such a database approach has been applied, for the first time in fusion science, towards a systematic study of the frequency spectra computed from reflectometry signals in the Tore Supra database, which are related to density fluctuations. In particular, decomposition of reflectometry frequency spectra has allowed to discover and quantify general trends of spectral characteristics (fractional contribution, width) for different components in Ohmic and low-confinement mode (L-mode) plasmas. In Ohmic plasmas, the contribution of the broadband component $\left(E_{\mathrm{BB}}\right)$ to the total spectrum power in the saturated Ohmic confinement (SOC) regime is observed to be systematically higher than in the linear Ohmic confinement (LOC) regime. A transition of the dominating instability from TEM to ITG, could explain the observed spectral modifications, which are supported by analysis of the dependence of density peaking on collisionality. The spectral characteristics of the broadband (BB) and low-frequency (LF) components were then investigated in L-mode plasmas with ICRH or LH heating. The similar trends of the BB components with collisionality observed in L-mode plasmas compared with the Ohmic cases suggests a similar explain by linking the frequency spectra to the underlying instabilities. The database analysis motivates more detailed studies by full-wave and gyrokinetic simulations, in order to confirm this link for both Ohmic and L-mode plasmas.

\section{INTRODUCTION}

Data science, involving methods from probability theory, statistics, machine learning, etc., is used extensively throughout science and engineering for learning patterns (clusters, trends) from data sets. Such patterns may reflect interesting underlying mechanisms of the system under study. While the value of these methods is in principle independent of the size of the data set, many applications target large databases with many variables. In fusion science, experiments generate large quantities of data and application of data science methods to fusion databases presents new opportunities with significant potential. The global energy confinement time ${ }^{1}$ or the power threshold for the transition from low to high confinement $^{2}$ are two well known examples of database analysis. Trends are often described in terms of other quantities through semi-empirical scaling laws. ${ }^{3,4}$ The parameters of these scaling laws, estimated using statistical regression methods, are instructive to the underlying turbulent transport properties and micro-instabilities. A database approach with statistical tools can be very useful for making an inventory of the characteristics of some aspects of the plasma, allowing investigations of systematic trends that could remain hidden in smallerscale studies. Although physical explanation of observed trends is not always straightforward, knowledge of trends

a)Electronic mail: yan.sun@ugent.be can drive the development of physical models, contribute to their verification and enable predictions.

A database approach is complementary to the more traditional shot-to-shot analysis, the latter involving a more strictly controlled range of plasma conditions and accurate measurements. The shot-to-shot analysis is usually supported by simulations to interpret the experimental observations, which in turn help to validate the simulation models and tools for dedicated discharges ${ }^{5-8}$. However, undertaking systematic simulations for even a part of a database may be hampered by prohibitive computing time and human resources. On the other hand, trends identified through a database analysis can lead to new experimental proposals and shot-to-shot analysis to explain or confirm the trends. For instance, in the dimensionless energy confinement time scaling law ${ }^{9}$, the dependence of confinement on the normalized Larmor radius can give clues on the Bohm or gyro-Bohm scaling of the turbulent eddies. This dependence has also been investigated through dedicated Larmor scaling experiments. ${ }^{10,11}$

Motivated by this spirit, the present study aims to characterize frequency spectra obtained by the core reflectometer for all shots performed on Tore Supra from 2002 to 2011, as well as trends of their characteristics. Reflectometry, a radar-like technique, can measure density profiles, as well as density fluctuations. More precisely, fixed-frequency reflectometry is a versatile and sensitive tool to characterize density fluctuations ${ }^{12-14}$.

In the majority of studies of trends in fusion science, the response variable is a single scalar quantity, such as the confinement time or L-H power threshold. How- 
ever, frequency spectra are characterized by multiple quantities, such as the spectrum shape, width, components, etc. On top of that, because of the complexity of the plasma-wave propagation physics, extracting the density fluctuation level from fixed-frequency measurement is not straightforward. ${ }^{15}$ It requires knowledge of the turbulence characteristic length, a parameter whose determination ask for extensive radial correlation length measurements. ${ }^{16}$ Therefore, in this database study, we necessarily have to restrict ourselves to studies of trends of the spectrum characteristics. In order to make the problem manageable, each spectrum has to be described using a sufficiently succinct model, that captures the essential characteristics of the spectrum. Since the frequency spectrum obtained by the Fourier transform algorithm requires several hundreds to a thousand frequency bins for a good spectral resolution, some sort of dimensionality reduction is needed, in order to decrease the number of values used to describe each spectrum.

Based on earlier work regarding frequency spectrum decomposition by Vershkov et al. ${ }^{17,18}$ and KrämerFlecken et $a .^{19,20}$, a parametrization method has been developed that reduces the number of parameters to describe the reflectometry power spectrum by two orders of magnitude ${ }^{21}$. This parametrization has allowed studies of dependencies of the reflectometry frequency spectra in Ohmic plasmas, distinguishing between the linear Ohmic confinement (LOC) and the saturated Ohmic confinement (SOC) regime. Furthermore, in this paper the effect of heating power and heating method (ion cyclotron resonance heating $(\mathrm{ICRH})$ or lower hybrid ( $\mathrm{LH})$ waves) has been investigated in L-mode plasmas.

The structure of this paper is as follows: Section II introduces the parametrization method of reflectometry spectra and recalls results obtained earlier in Ohmic discharges. After separating the Ohmic discharges into the LOC and SOC regimes, Section III discusses radial profiles of the broadband $(\mathrm{BB})$ contribution $\left(E_{\mathrm{BB}}\right)$ at different edge safety factor $\left(q_{\psi}\right)$ and the dependence of $E_{\mathrm{BB}}$ on collisionality. A possible interpretation of the observed differences between the LOC and SOC regimes is provided in terms of a transition of the dominant instability, supported by gyrokinetic simulations and confirmed by profiles of the width of the low-frequency (LF) spectral component $\left(W_{\mathrm{LF}}\right)$. Section IV extends the analysis to $\mathrm{LH}$ and ICRH plasmas at different heating power and $q_{\psi}$, also discussing the BB width $\left(W_{\mathrm{BB}}\right)$. Finally, conclusions and discussions regarding future work are presented in Section V.

\section{REFLECTOMETRY FREQUENCY SPECTRUM PARAMETRIZATION METHOD}

\section{A. Decomposition and fit of frequency spectrum}

The details of the data analysis techniques used to parametrize the fluctuation frequency spectra from core

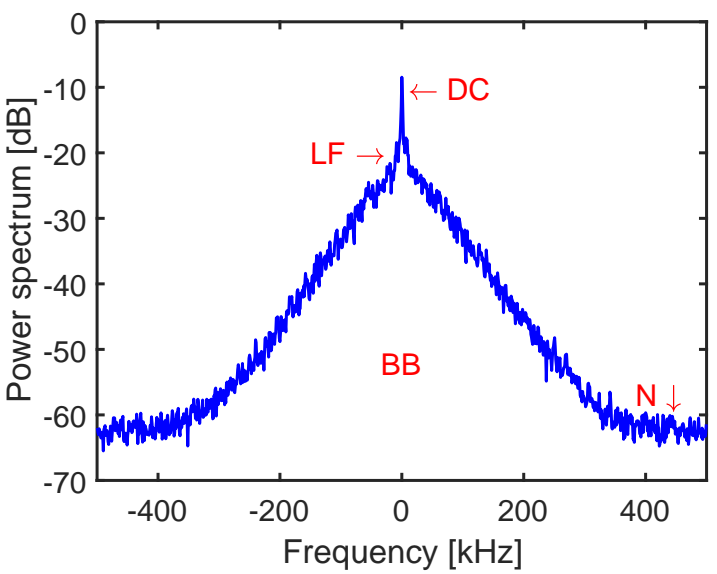

Figure 1. A typical spectrum with four components: the direct current component (DC), the low-frequency fluctuations $(\mathrm{LF})$, the broadband turbulence $(\mathrm{BB})$, and the noise level $(\mathrm{N})$.

reflectometry ${ }^{22}$ can be found in Ref. 21 . We briefly recall the main aspects here. First, for each fixed frequency plateau, the frequency spectrum is computed using a Welch's power spectral density method. To compare the results across the whole database, each spectrum is normalized to its total power. The total (reflected) power can indeed vary by more than five orders of magnitude, even at fixed radial positions. This large amplitude variation can be caused not only by the variability in the reflectometer efficiency (output power and conversion loss) with the probing wave frequency, but also by modification of the poloidal and possibly toroidal curvature of the cutoff layer, which can induce dramatic amplitude variations of the signal reflected back to the receiving antenna.

Next, the frequency spectrum is decomposed into four components: the direct current component (DC), the low-frequency fluctuations (LF), the broadband turbulence $(\mathrm{BB})$, and the noise level $(\mathrm{N})$, as shown in Figure 1. Each component is then fitted by a single parameterized function. A constant value quantifies the noise level. Two Gaussian functions are used for the LF and DC components, whose both peaks are close to the central (zero) frequency (Figure 1). The fit of the BB turbulence is more complicated, as the $\mathrm{BB}$ component can exhibit a broader variety of spectral shapes, compared to the other components. Both the Taylor ${ }^{23}$ and generalized Gaussian model have been shown to provide excellent fitting performance, each using two parameters for the BB spectral shapes. The interpretation of the Taylor model is more meaningful from a physical point of view. ${ }^{23}$ Moreover, the generalized Gaussian model can sometimes have a peaked shape for the $\mathrm{BB}$ component. In turn, this may lead to an overestimation of the BB contribution to the total spectrum and instability of the algorithm to fit the BB width. Therefore, the spectral parameters in this study are those obtained from the Taylor model.

In order to capture simultaneously the features of the 


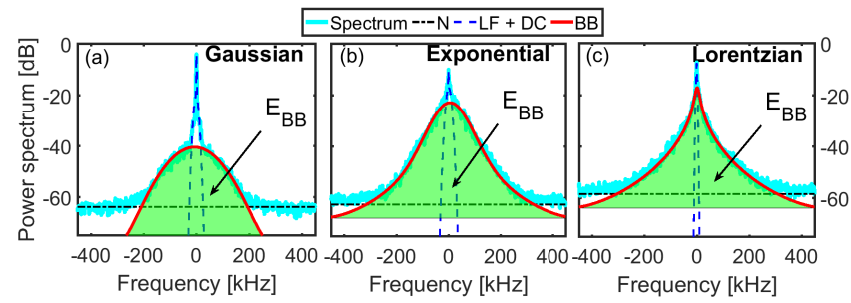

Figure 2. Fit of the different components of typical reflectometry spectra with varying shapes: approximately (a) Gaussian, (b) double exponential (triangular on the logarithmic scale) and (c) Lorentzian. The DC and LF components were fitted by two Gaussian functions, the noise level (N) by a constant and the BB component by the FFT of the Taylor function. The green shaded area represents the integrated $\mathrm{BB}$ contribution, denoted by $E_{\mathrm{BB}}$.

low-frequency and high-frequency part of the frequency spectra, the fitting cost function relies on both the linear and logarithmic scale, attributing an equal weight factor in this study. Furthermore, multiple initial guesses were used to ensure global convergence of the fitting (optimization) process. The fitting results using the Taylor model for the three typical BB spectral shapes(approximately) (a) Gaussian, (b) double exponential (Laplacian) and (c) Lorentzian (Cauchy) - are depicted in Figure 2.

Other components (Alfvén modes, modes associated to sawteeth, quasi-coherent modes, etc.) that are not taken into account for the fitting, represent only a small fraction of the spectrum energy, as their bandwidth is limited and the amplitude is usually moderate compared to the BB or the LF components. The impact of these narrow band modes on the fit is also weak, since the fit is carried out in linear and logarithmic scale simultaneously. Extra components might be added in future work, to fit also the quasi-coherent (QC) modes, enabling systematic studies of their dependencies. ${ }^{24}$

Our parametrization technique of the frequency spectrum drastically reduces the number of parameters needed to describe, quantify and compare the various spectra, by two orders compared to the number of values typically produced by a Fourier transform algorithm. Each spectrum component is described by a handful of parameters: the amplitude, the position, the width and, for the BB component, the shape. More precisely, the following quantities are used to investigate the evolution of the reflectometry frequency spectra across the database. The $\mathrm{BB}$ contribution $\left(E_{\mathrm{BB}}\right)$ in each frequency spectrum is calculated by integrating the BB component, as shown in Figure 2. $E_{\mathrm{BB}}$ ranges between 0 and 1 due to the normalization by the total power of the corresponding spectrum. We assume that the scattered electric fields due to the $\mathrm{BB}$ turbulence contain the same information whatever the amplitude of the reflected signal. When small, $E_{\mathrm{BB}}$ is proportional to the phase fluctuation level induced by the BB turbulence, and thus to the BB den- sity fluctuation level. However, when the normalized $E_{\mathrm{BB}}$ is close to one, the previous assumption becomes invalid, as the scattered fields due to the BB turbulence at the cutoff layer become stronger than the electric fields associated to the cutoff reflection process. In other words, the normalized $E_{\mathrm{BB}}$ can be assumed to be proportional to the density fluctuation level when the normalized $E_{\mathrm{BB}}$ is small $(\ll 1)$, whereas this assumption becomes invalid when the normalized $E_{\mathrm{BB}}$ is close to one. For simplicity, hereafter we refer to the normalized $E_{\mathrm{BB}}$ as $E_{\mathrm{BB}}$. Additional spectral properties can also be obtained from the parametrization, e.g. the standard deviation, i.e. the square root of the second central moment of the $\mathrm{BB}$ or the LF component, which we refer to as the BB width $\left(W_{\mathrm{BB}}\right)$ or the LF width $\left(W_{L F}\right)$.

This paper studies general trends of these parameters, which reflect various aspects of the spectral properties of different components (BB and LF), focusing in particular on the $\mathrm{BB}$ contribution $E_{\mathrm{BB}}$.

\section{B. Database and application}

The parametrization method was applied to all fixedfrequency measurements performed with the core fluctuation reflectometer that operated on Tore Supra from 2002 to 2011. This reflectometer covers the band from 105 to $155 \mathrm{GHz}$ in X-mode. Most of the time, it offered two independent channels that performed simultaneous measurements at two different positions. The lowest frequency (105 to $115 \mathrm{GHz}$, depending on the channel), always corresponds to a position inside the plasma. At low to moderate frequencies, the first cutoff positions were at around mid-radius. The first cutoff positions move outward with increasing central electron density. At moderate to high magnetic fields $(B>3.5 \sim 4 \mathrm{~T})$ and not too high central electron density $\left(n_{\mathrm{e}}(0)<7 \times 10^{19} \mathrm{~m}^{-3}\right)$, the reflectometer accessibility extends beyond the plasma center inside the gradient zone almost up to the plasma edge. $^{22}$

The database includes more than 350,000 reflectometry fluctuation measurements from about 6,000 discharges. Each measurement lasts typically $\sim 10 \mathrm{~ms}$ and various plasma parameters were calculated by averaging over each of these time windows. The 6,000 discharges contain a large number of Ohmically heated plasmas, as well as plasmas with auxiliary heating: ICRH, LH and a limited number with ECRH. The present database includes near 100 parameters that fall into three categories:

- The global operational parameters: the on-axis toroidal magnetic fields $\left(B_{t, 0}\right)$, the plasma current $\left(I_{p}\right)$, the line-integrated density $\left(n_{l}\right)$, the edge safety factor $\left(q_{\psi}\right)$, the major radius $(R)$, the minor radius $(a)$, the heating power for different scenarios ( $\left.P_{\text {Ohmic }}, P_{\mathrm{ICRH}}, P_{\mathrm{LH}}, P_{\mathrm{ECRH}}\right)$, etc.

- The local plasma parameters: the cutoff position $r_{c}$ and the electron density $n_{\mathrm{e}}(r)$ from interferometry 
measurements, the electron temperature $T_{\mathrm{e}}(r)$ from electron cyclotron resonance (ECE) measurements, as well as the gradients of density, temperature and refractive index at the cutoff positions.

- The 11 spectrum decomposition parameters and additional spectrum characteristics: the total power of each spectrum, the BB contribution $\left(E_{\mathrm{BB}}\right)$, the BB width $\left(W_{\mathrm{BB}}\right)$, the LF width $\left(W_{\mathrm{LF}}\right)$, etc.

A few more points need to be mentioned before addressing the actual database analysis. First, unreliable measurements have been excluded from the database. More precisely, spectra with too low signal-to-noise ratio $(<25 \mathrm{~dB})$ have been removed from the analysis. Spectra with large Doppler effect (central shift $>50 \mathrm{kHz}$ ) have also been excluded, since in these cases the physical process and the probed wavenumber are different from the standard reflectometry measurement signals. Specifically, the standard measurement signals come from the perpendicular reflection of the probing waves at the cutoff positions, and therefore reflect the local fluctuations with very low wavenumber. Large Doppler shift results from backscattering at finite wavenumber. This backscattering could appear when the magnetic axis is not on the equatorial plane, causing the reflectometer line-of-sight to be not normal to the cutoff layer surface, or when the turbulent structures are tilted with respect to the poloidal direction. Toward the edge, the tilting of the reflective surface in the toroidal direction due to the magnetic field ripple can also lead to strong Doppler shift. Second, before further analysis, only measurements performed during steady-state conditions have been studied. For example, the reflectometer acquisitions during the current or power ramp-up or ramp-down phase have been filtered out to avoid additional complexity.

The present study considers the complete radial extent of the plasmas, except the edge region at the low-fieldside (LFS), where the density fluctuation level $(\delta n / n)$ reaches $\sim 10 \%$ and nonlinear effects may complicate the analysis. ${ }^{25,26}$ Furthermore, spectra in the radial range at the LFS near the edge $(0.6<\rho<1$, with $\rho=r / a$ the normalized radius) are more prone to the large Doppler shift partly caused by the high ripple ( $6 \%$ at the plasma edge) of the magnetic field. Finally, this part of the plasma cannot be probed at low density due to the reflectometer frequency range. This condition occurs for the low density Ohmic discharges and most LH plasmas. For these reasons, the number of valid measurements is significantly reduced beyond $\rho>0.6$, rendering estimation of trends unreliable. In practice, the radial range was set to $-1<\rho<0.6$. Here, $\rho=0$ denotes the plasma center and negative $\rho$ corresponds to the HFS.

In a first analysis of the database, radial profiles were investigated of the broadband contribution $\left(E_{\mathrm{BB}}\right)$ to the frequency spectra at different edge safety factor $q_{\psi}$ in Ohmic discharges. ${ }^{21}$ Figure 3 shows one example of the radial profiles of $E_{\mathrm{BB}}$ resulting from $\sim 30,000$ measure-

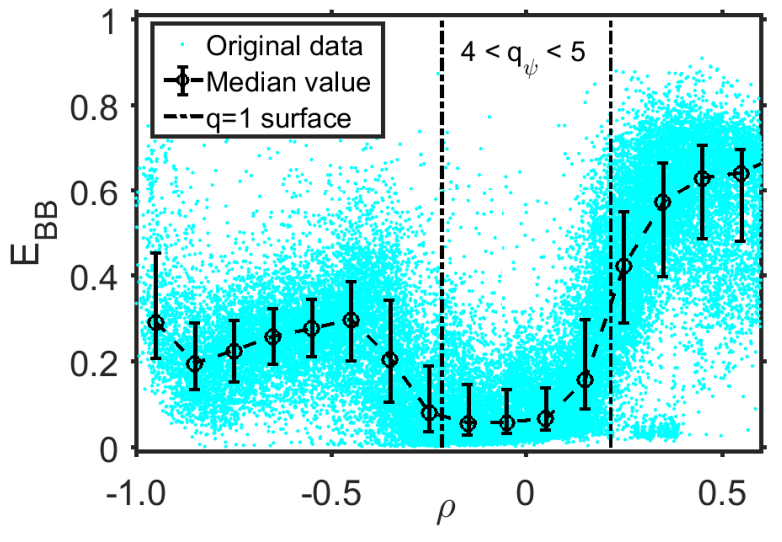

Figure 3. Radial profile of the $\mathrm{BB}$ contribution $\left(E_{\mathrm{BB}}\right)$ with $4<q_{\psi}<5$ in Ohmic plasmas. The approximate positions of the $q=1$ surface obtained from the empirical scaling $\rho_{q=1} \approx$ $1 / q_{\psi}{ }^{27}$ are shown.

ments (cyan points) in $\sim 1,400$ Tore Supra discharges under Ohmic conditions and with $4<q_{\psi}<5$. A drop of $E_{\mathrm{BB}}$, which we refer to as the $E_{\mathrm{BB}}$ basin, was observed in the core region. This drop of density fluctuation inside the $q=1$ surface was also observed before ${ }^{25}$. Database analysis shows that the location and width of the $E_{\mathrm{BB}}$ basin are linked to the $q=1$ surface. Moreover, there is a strong asymmetry between the HFS and LFS, as $E_{\mathrm{BB}}$ is generally higher at the LFS.

Figure 3 clearly shows the considerable dispersion in the data at fixed radial positions, as the measurements were taken under a broad variety of plasma conditions. Therefore, in order to better reflect the trends with radius, the local median obtained from narrow radial intervals $(\delta \rho= \pm 0.05)$ is also displayed in Figure 3. The error bars are given by the mean absolute deviation around the median values within each interval. The same analysis methods will be used for all profile studies of spectra parameters in the remainder of this paper.

\section{FREQUENCY SPECTRUM EVOLUTION IN DIFFERENT CONFINEMENT REGIMES OF OHMIC PLASMAS}

\section{A. Empirical confinement regime determination}

We have investigated in what way the different Ohmic confinement regimes, i.e. the linear Ohmic confinement (LOC) regime and the saturated Ohmic confinement (SOC) regime, affect the frequency spectra. The density threshold of the LOC-SOC transition can be approximated as: ${ }^{28}$

$$
n_{\mathrm{LOC}-\mathrm{SOC}} \approx \frac{5 I_{p} \mu_{0}}{\pi a^{2}} \sqrt{\frac{A_{i} \kappa}{2}} .
$$

Here, $n_{\mathrm{LOC}-\mathrm{SOC}}$ is the central line-averaged density in $10^{19} \mathrm{~m}^{-3}, I_{p}(\mathrm{~A})$ the plasma current, $a(\mathrm{~m})$ the minor 
radius, $A_{i}$ the atomic mass number and $\kappa$ the elongation. A previous study based on a number of dedicated Tore Supra discharges (Ref. 24) shows that (1) tends to overestimate the density threshold of the LOC-SOC transition. However, for Tore Supra, all prediction parameters in the scaling (1), except $I_{p}$, are almost constant. Specifically, Tore Supra had a circular cross-section and operated with a bottom limiter at an almost constant vertical position, ensuring $\kappa \approx 1$ and $a \approx 0.72 \mathrm{~m}$. Both $\kappa$ and $a$ have uncertainties less than $5 \%$. With deuterium operation the atomic mass number $A_{i}$ is 2 , while impurity concentrations were limited, usually less than $10 \%$.

The above considerations have motivated a reduced empirical scaling law for $n_{\mathrm{LOC}-\mathrm{SOC}}$ in terms of $I_{p}$, making use of a large number of discharges. Figure 4 describes the method to obtain the empirical scaling law of $n_{\mathrm{LOC}-\mathrm{SOC}}$. At fixed plasma current in Ohmic plasmas, the total energy confinement time increases with the central line-averaged density, passing a knee point at the approximate threshold density and then reaching saturation. Figure 4 (a) shows such an example in the database for $I_{p}=0.8 \mathrm{MA}$. To compensate for the scatter of the data, the median confinement time is calculated in small density ranges. The knee point of the median curve then provides the estimate of the LOC-SOC transition density threshold. Applying this method at different $I_{p}$, the following scaling relation could be obtained, depicted in Figure 4 (b):

$$
n_{\mathrm{LOC}-\mathrm{SOC}}^{\mathrm{TS}} \approx 2.6 \times I_{p},
$$

with $n_{\mathrm{LOC}-\mathrm{SOC}}$ in $10^{19} \mathrm{~m}^{-3}$ and $I_{p}$ in MA. The LOCSOC transition densities obtained from two dedicated density scans at $I_{p}=0.5 \mathrm{MA}$ and $1.2 \mathrm{MA}^{24}$ conform with the scaling law (2) obtained from the Tore Supra database.

Next, the central line-averaged density $n_{l, 0}$ from interferometry measurements ${ }^{29}$ was used to determine the confinement regime for each reflectometer acquisition, assuming a $\pm 10 \%$ uncertainty on the threshold given by the scaling law:

- LOC: $n_{l, 0}<0.9 \times n_{\mathrm{LOC}-\mathrm{SOC}}^{\mathrm{TS}}$;

- SOC: $n_{l, 0}>1.1 \times n_{\mathrm{LOC}-\mathrm{SOC}}^{\mathrm{TS}}$.

In the remainder, we refer to the intermediate region around the density threshold as the LOC-SOC transition regime.

\section{B. Dependence of $E_{\mathrm{BB}}$ on confinement regime}

Equipped with a criterion for discriminating between the LOC and SOC regimes, the radial profiles of $E_{\mathrm{BB}}$ can be studied separately in those regimes. In both cases, the radial profile of $E_{\mathrm{BB}}$ exhibits similar general characteristics as shown in Figure 3. However, specific differences of $E_{\mathrm{BB}}$ between the LOC and SOC regimes are difficult
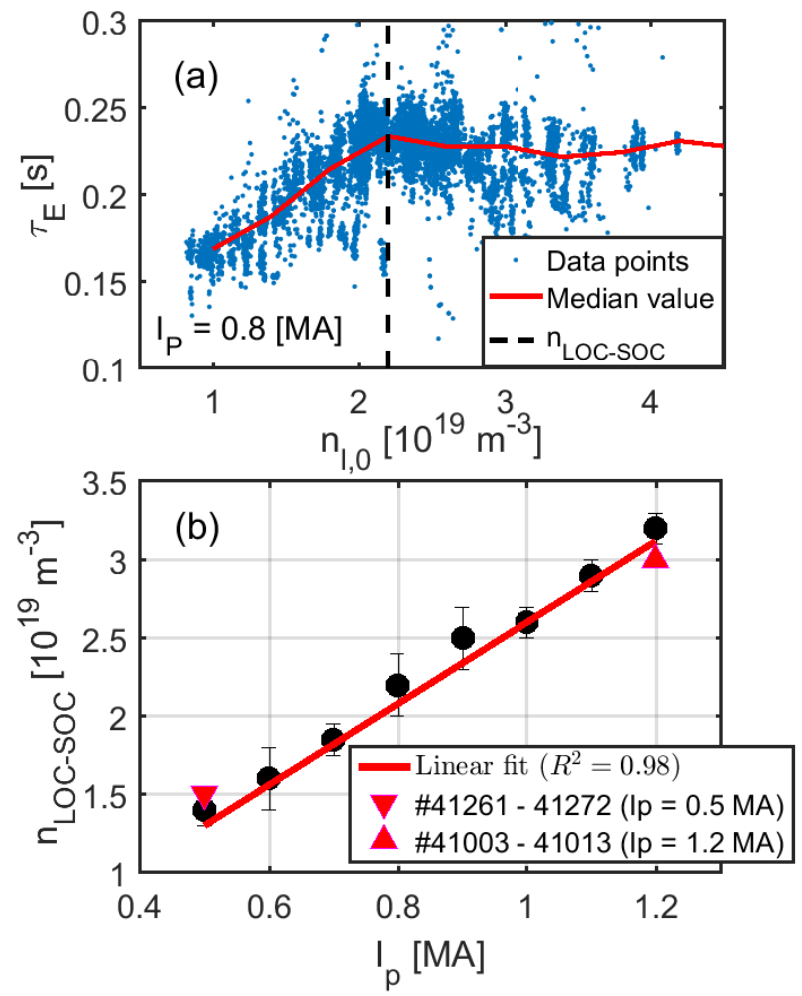

Figure 4. (a) Evolution of the global confinement time with central line-averaged density at $I_{p}=0.8 \mathrm{MA}$. (b) Empirical scaling law of the LOC-SOC transition density threshold $\left(n_{\mathrm{LOC}-\mathrm{SOC}}\right)$ in terms of plasma current $\left(I_{p}\right)$.

to deduce from the original profiles, since many spectra from both regimes are overlapping. Therefore, we plot the radial profiles of the local median of $E_{\mathrm{BB}}$ in both regimes, as shown in Figure 5. The typical evolution of $E_{\mathrm{BB}}$ across the radial extent of the plasma, as well as the $E_{\mathrm{BB}}$ basin in the central region, are again observed in both LOC and SOC regimes, for all $q_{\psi}$ ranges. The analysis also recovers the link between the basin (width, location) and the $q=1$ surface. Far outside the $q=1$ surface at the LFS, $E_{\mathrm{BB}}$ approaches 1 in both regimes, i.e. with most of the energy in the $\mathrm{BB}$ component, whereas at the HFS $E_{\mathrm{BB}}$ remains at a moderate level. Furthermore, $E_{\mathrm{BB}}$ in SOC is systematically higher than in LOC throughout the plasma cross-section for all $q_{\psi}$. Specifically, for LOC inside the $q=1$ surface, $E_{\mathrm{BB}}$ is very low, especially at low $q_{\psi}$. This means that for LOC in the very core region $(\rho \sim 0)$ only a minor part of the energy is in the BB component, or equivalently, most of the energy in the reflectometry spectra is in the LF component. In contrast, in the $\mathrm{SOC}$ regime $E_{\mathrm{BB}}$ can still reach levels of $20 \%$ at $\rho \sim 0$. 

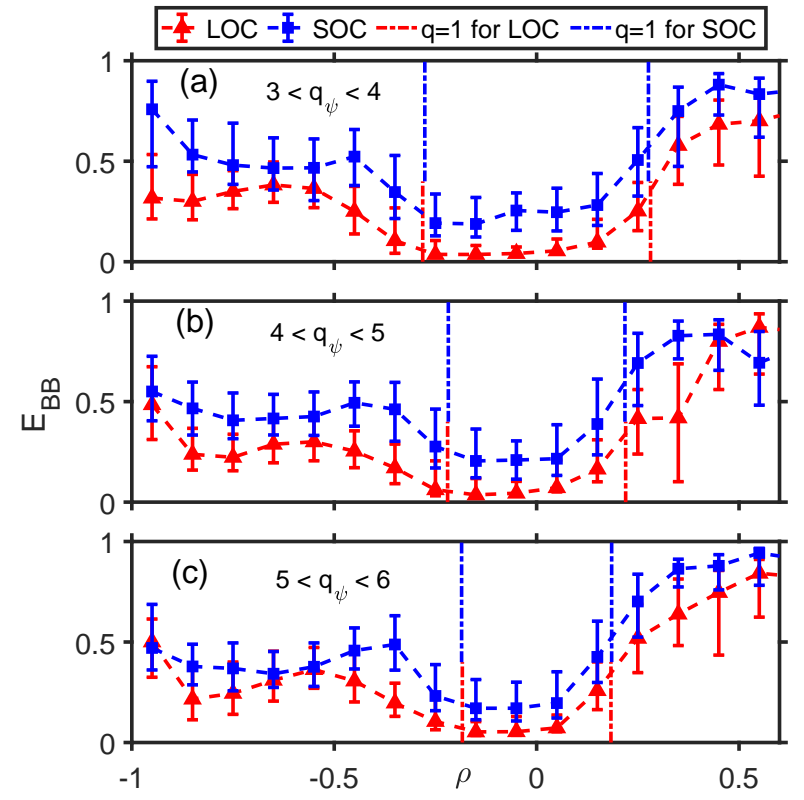

Figure 5. Median radial profiles of $E_{\mathrm{BB}}$ for different $q_{\psi}$ in the LOC and SOC regimes. The approximations of the $q=1$ positions were obtained separately for both regimes by the same method as in Figure 3.

\section{Evolution of density peaking with collisionality}

The transition from the LOC to SOC confinement regime has been attributed in previous studies to a change in the dominant instability from trapped electron modes (TEM) to ion temperature gradient (ITG) modes. ${ }^{30,31}$ Experimentally, this connection between confinement regime and dominant instability has also been studied through the dependence of density peaking and intrinsic rotation on collisionality in various devices, such as Alcator C-Mod and ASDEX Upgrade. ${ }^{32,33}$ Collisions have a crucial impact on the trapped particle modes via the detrapping mechanism, i.e. collisions tend to stabilize TEM modes.

Among various definitions of collisionality, the effective collisionality $\left(\nu_{\text {eff }}\right)$ for drift wave instabilities has been adopted as an excellent indicator for TEM stabilization. It is defined as the ratio between the electronion collision frequency and the curvature drift frequency: $\nu_{\text {eff }}=\nu_{\mathrm{ei}} / \omega_{\mathrm{De}}{ }^{34,35}$ For ITG and TEM instabilities, the curvature drift frequency provides an estimate of the mode growth rate, and is defined as $\omega_{\mathrm{De}}=2 k_{\perp} \rho_{L} c_{s} / R$, with $k_{\perp}$ the perpendicular wave number, $\rho_{\mathrm{L}}$ the ion Larmor radius, $R(\mathrm{~m})$ the major radius and $c_{\mathrm{s}}$ the ion acoustic velocity. Accordingly, $\nu_{\text {eff }}$ has been approximated as follows: ${ }^{34,36}$

$$
\nu_{\mathrm{eff}} \approx 0.1 R Z_{\mathrm{eff}} n_{\mathrm{e}} T_{\mathrm{e}}^{-2}
$$

where $Z_{\text {eff }}$ is the effective charge number, $n_{\mathrm{e}}\left(10^{19} \mathrm{~m}^{-3}\right)$ the electron density and $T_{\mathrm{e}}(\mathrm{keV})$ the electron temperature. In this approximation, the normalized perpendic-

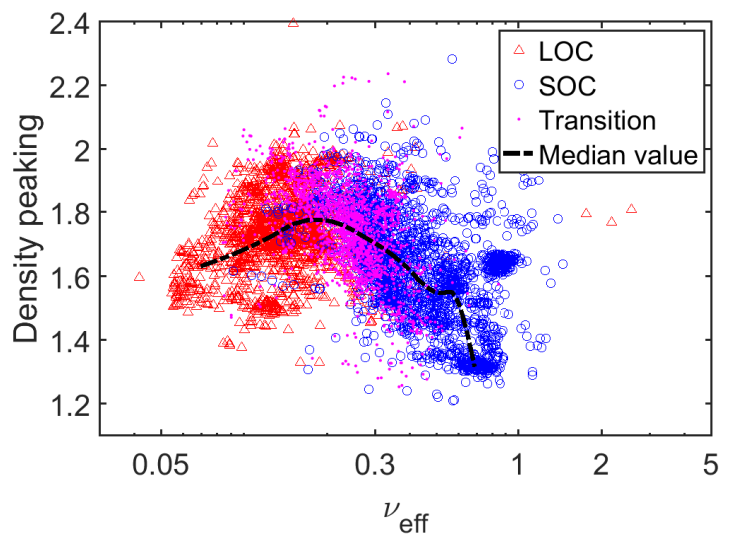

Figure 6. Evolution of the density peaking factor with respect to the effective collisionality for Ohmic plasmas with $3<q_{\psi}<$ 6.

ular wave number $k_{\perp} \rho_{L}$ has been estimated to be $\sqrt{0.1}$, which is the characteristic value for core density fluctuations and within the capability of reflectometers. Since the local $Z_{\text {eff }}$ is unavailable for the Tore Supra database, the integrated (tangential) $Z_{\text {eff }}$ value has been used in calculating $\nu_{\text {eff }}$. Although some systematic uncertainty of $\nu_{\text {eff }}$ might occur when a constant $Z_{\text {eff }}$ is used, global trends at fixed radial positions are not noticeably affected, especially in the core region.

Figure 6 shows the evolution of the density peaking with respect to the effective collisionality $\nu_{\text {eff }}$ for a broad range of edge safety factor $\left(3<q_{\psi}<6\right)$ in the central region $(-0.1<\rho<0.1)$. The density peaking has been defined as the ratio between the central density and the averaged density, both from interferometry measurements. In addition, the central density and temperature have been used in calculating $\nu_{\text {eff }}$. Since a strong dispersion of data occurs at fixed $\nu_{\text {eff }}$, the smoothed median values at different $\nu_{\text {eff }}$ are shown. The median values have been calculated from a small fixed interval ( 0.01) of $\nu_{\text {eff }}$ in the logarithmic scale.

As expected, the LOC and SOC regimes correspond to the low and high ranges of $\nu_{\text {eff }}$, respectively. In the LOC regime, the density peaking increases with $\nu_{\text {eff }}$, whereas in the SOC regime the density peaking decreases. These opposite trends result in a maximal density peaking factor for the transition regime, at about $\nu_{\text {eff }} \sim 0.2$. At high $\nu_{\text {eff }}$, the decrease of the density peaking with $\nu_{\text {eff }}$ has been widely studied on ASDEX Upgrade. ${ }^{30,34}$ At low $\nu_{\text {eff }}$, the increase of the density peaking might be explained by the impact of the plasma resistivity on the neoclassical Ware pinch due to the trapped particles. Since the plasma resistivity $\left(\propto T_{e}^{-3 / 2}\right)$ increases with the effective collisionality $\left(\propto T_{e}^{-2}\right)$, the increase of the effective collisionality $\left(\nu_{\text {eff }}\right)$ then leads to an increase of the toroidal electric field $\left(E_{\phi}\right)$. Furthermore, this increase of $E_{\phi}$ induces an increase of the (inward) Ware pinch, resulting in a higher density peaking. With a further increase of $\nu_{\text {eff }}$, leading 


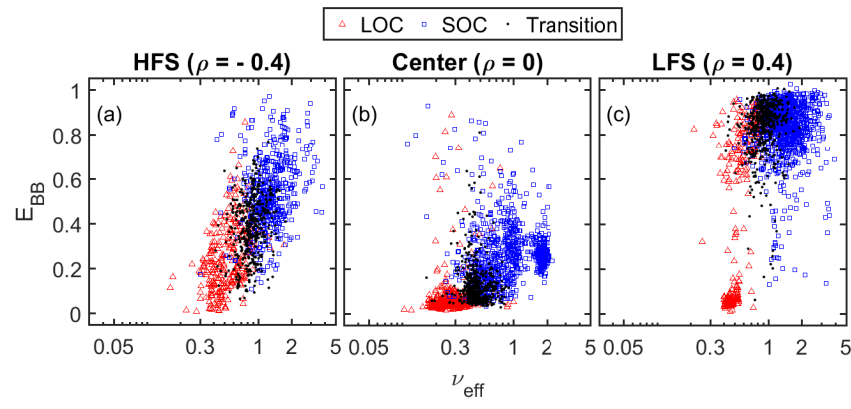

Figure 7. Evolution of the broadband contribution $\left(E_{\mathrm{BB}}\right)$ with effective collisionality $\left(\nu_{\text {eff }}\right)$ in the LOC, SOC and transition regimes at (a) the HFS $(\rho=-0.4)$, (b) the plasma center inside the basin $(\rho=0)$ and (c) the LFS $(\rho=0.4)$. Note the logarithmic scale for $\nu_{\text {eff }}$.

to transition from the banana to the plateau regime, one could expect a saturation or even an attenuation of the Ware pinch, due to the stabilization of trapped particles (TEM instability). The density peaking is highest at the transition regime between the LOC and SOC regimes. It could be a signature of the transition from TEM to ITG dominated turbulence.

This database observation with hundreds of Ohmic shots confirms the results of a previous detailed analysis of an Ohmic discharge with an $I_{p}$ scan (Tore Supra \#48102), supported by GENE gyrokinetic simulations. Linear simulations showed that the TEM instability was destabilized at high $I_{p}(\mathrm{LOC})$ and stabilized at low $I_{p}$ (SOC). ${ }^{24,37,38}$

\section{Evolution of the BB amplitude with the effective collisionality}

Since the BB contribution is higher in SOC compared to LOC, as shown by Figure 5, and the transition from LOC to SOC occurs when scanning the effective collisionality, one would expect a trend of the BB contribution with collisionality. Indeed, Figure 7 shows the dependence of $E_{\mathrm{BB}}$ on $\nu_{\mathrm{eff}}$ in the LOC, SOC and transition regimes at the HFS $(\rho=-0.4)$, the plasma center $(\rho=0)$ and the LFS $(\rho=0.4)$. The HFS and LFS radial positions $(\rho= \pm 0.4)$ have been chosen to be neither in the region with large radial changes of $E_{\mathrm{BB}}(0.2<|\rho|<0.3)$, nor in the saturation region $(\rho>0.6)$ (see Figure 5). At each $\rho$, the data include all the $q_{\psi}$ ranges shown in Figure 5. Note that $\nu_{\text {eff }}$ was estimated from the local values of $n_{\mathrm{e}}$ and $T_{\mathrm{e}}$. For $Z_{\text {eff }}$, again the estimate from a tangential line of sight was used to calculate $\nu_{\text {eff }}$. It is strongly weighted by the plasma core, so even if $Z_{\text {eff }}$ would increase toward the edge, all the data points at the LFS and HFS would merely shift slightly toward higher $\nu_{\text {eff }}$ (under the assumption that the shape of the $Z_{\text {eff }}$ profile does not change drastically with increasing $\nu_{\text {eff }}$ ).

Despite the significant scatter of $E_{\mathrm{BB}}$ in the plots, the following observations can be made. Inside the central
$E_{\mathrm{BB}}$ basin (Figure $7(\mathrm{~b})$ ), in the LOC regime, $E_{\mathrm{BB}}$ remains low $(\sim 0.1)$ for $\nu_{\text {eff }}$ ranging from below 0.1 to around 0.3. Above $\nu_{\text {eff }}=0.3, E_{\mathrm{BB}}$ generally increases rapidly as the plasma transits from the LOC to the SOC regime. Outside the basin (Figure 7 (a) and (c)), $\nu_{\text {eff }}$ (calculated from local $n_{\mathrm{e}}$ and $T_{\mathrm{e}}$ ) is generally higher than in the center, due to the stronger dependence of $\nu_{\text {eff }}$ on $T_{\mathrm{e}}$ than $n_{\mathrm{e}}$ (from (4): $\nu_{\mathrm{eff}} \propto T_{\mathrm{e}}^{-2}$ and $\left.\nu_{\mathrm{eff}} \propto n_{\mathrm{e}}\right)$ and a $T_{\mathrm{e}}$ profile that is steeper compared to the $n_{\mathrm{e}}$ profile. At the HFS (Figure 7 (a)), $E_{\mathrm{BB}}$ increases almost linearly when $\nu_{\text {eff }}$ changes from 0.3 to 3 on a logarithmic scale. At the LFS (Figure 7 (c)), $E_{\mathrm{BB}}$ also increases with $\nu_{\text {eff }}$, but it quickly reaches values close to saturation when $\nu_{\text {eff }}>0.5$. Note that a cluster of spectra accumulates at $\nu_{\text {eff }} \sim 0.5$ when $E_{\mathrm{BB}}<0.2$. This remarkable cluster corresponds to the cluster in Figure 3 (low $E_{\mathrm{BB}}$ at the LFS). A preliminary investigation has identified that the spectra forming that cluster correspond to a number of contiguous Tore Supra discharges (\#40481 - \#40490). These measurements come from the same acquisition channel and have the same probing frequency $(120 \mathrm{GHz})$, indicating a possible technical problem of this channel for this series of discharges.

Although here the results at only three radial positions are presented, similar trends are observed at other radial positions, except close to the plasma edge, where saturation occurs. The saturation could be due to an increase of the fluctuation level close to the plasma edge in combination with stronger nonlinear effects. In summary, an increasing trend of $E_{\mathrm{BB}}$ with $\nu_{\mathrm{eff}}$ is consistently observed throughout the entire plasma cross-section. Inside the $E_{\mathrm{BB}}$ basin, at low $\nu_{\mathrm{eff}}$ the increase is relatively weak, becoming steeper above $\nu_{\text {eff }}=0.3$. In addition, we have investigated the evolution of the BB width and shape with $\nu_{\text {eff }}$, but the dependence is complicated and only weak trends could be identified. Additional global plasma parameters will need to be considered to extract general trends for these two parameters.

\section{E. Interpretation of spectral changes across the LOC-SOC transition}

A possible interpretation of the observed trends of the BB component in terms of $\nu_{\text {eff }}$ could be related to a change of the dominant instability from TEM to ITG, when crossing from the LOC to the SOC regime. This would correspond with the evolution of the density peaking with collisionality in Figure 6. Further support to this hypothesis is lent by an earlier, dedicated study of the effect of the dominant instability on the density fluctuation spectra by gyrokinetic simulations in the Ohmic Tore Supra discharge \#48102. ${ }^{38,39}$ The simulations were performed at $\rho=0.37$, which is close to the position in the database study $(\rho=0.4)$ at the LFS. Figure 8 shows that, in the LOC regime when TEM dominates, the $\mathrm{BB}$ component is separated from a narrow LF component, whereas in the SOC regime when ITG dominates, a 


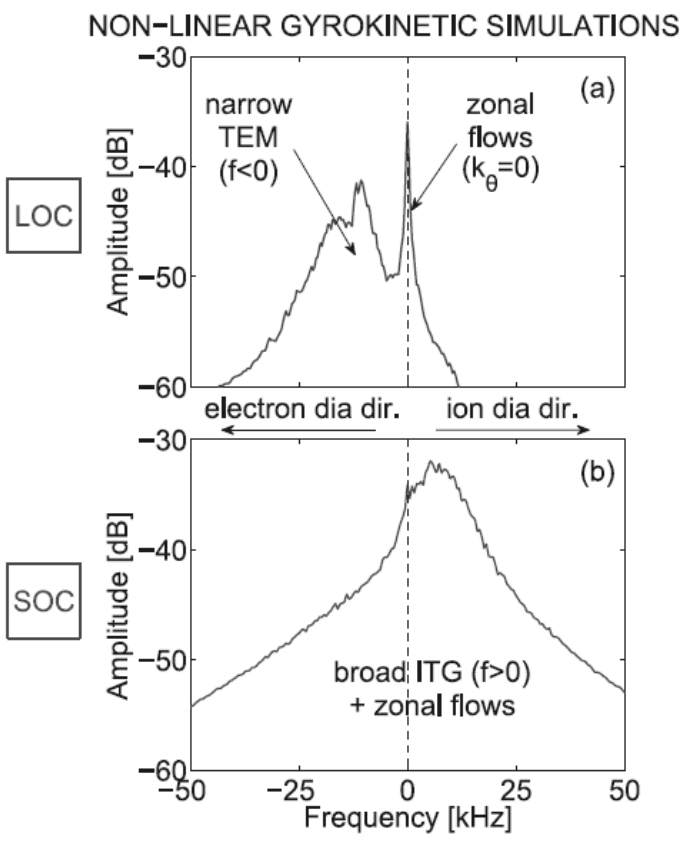

Figure 8. Density fluctuation spectra by gyrokinetic simulations in Tore Supra discharge \#48102 at $\rho=0.37$ for (a) the LOC and (b) the SOC regime. Reprinted from Ref. 39, copyright owned by IOP Publishing.

much wider BB component exists and the LF component merges into the $\mathrm{BB}$ component.

To support this interpretation, we have studied the radial profiles of the LF width $\left(W_{\mathrm{LF}}\right)$ in the LOC and SOC regimes, as shown in Figure 9. It is clear that $W_{\mathrm{LF}}$ in the SOC regime is systematically higher than in the SOC regime at all radial positions. However, the estimate of $W_{\mathrm{LF}}$ from our parametrization technique might be less reliable when the BB contribution is high. From Figure 5 , outside the central basin, i.e. outside the $q=1$ surface, $E_{\mathrm{BB}}$ may reach a value of 0.5 (HFS) or close to 1 (LFS). Accordingly, when $E_{\mathrm{BB}}$ is higher than 0.5, most of the energy is in the $\mathrm{BB}$ component and the LF component disappears or merges with the $\mathrm{BB}$ component, rendering the fit of the LF component, and thus $W_{\mathrm{LF}}$, unstable. Therefore, we focus only on the central region where $E_{\mathrm{BB}}$ is far from saturation and thus $W_{\mathrm{LF}}$ is more reliable. At the center inside the $q=1$ surface, the LF width in the SOC regime $(\sim 8 \mathrm{kHz})$ is approximately two times the width in the LOC regime $(\sim 4 \mathrm{kHz})$. The evolution of $W_{\mathrm{LF}}$ with the effective collisionality has also been investigated, but there is only a weak dependence of $W_{\mathrm{LF}}$ on $\nu_{\text {eff }}$. Other parameters might be needed to get clearer trends of $W_{\mathrm{LF}}$.
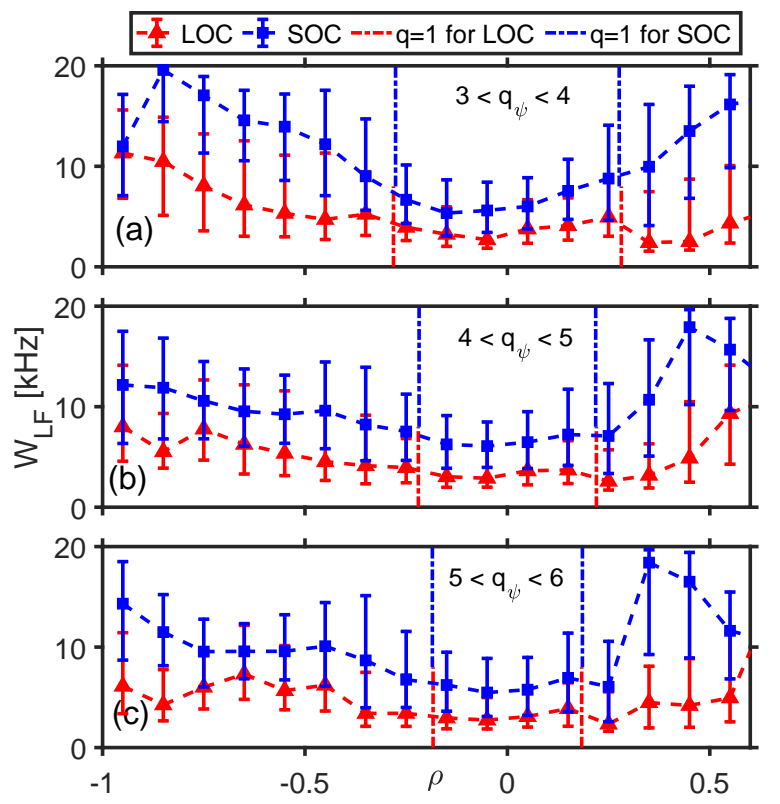

Figure 9. Median radial profiles of the LF width $W_{\mathrm{LF}}$ for different $q_{\psi}$ in the LOC and SOC regimes.

\section{FREQUENCY SPECTRUM IN PLASMAS WITH AUXILIARY POWER}

Having investigated the Ohmic plasmas, we now turn to the case with additional heating power. In Tore Supra, ICRH and LH are the two most commonly applied methods for auxiliary heating or current drive. Note that most of the Tore Supra discharges with LH were devoted to current drive, which allows the existence of fast electrons and thus varying ratios of trapped electrons. For clarity, we focus on plasmas that are either pure ICRH or LH plasmas.

\section{A. Radial profiles of $E_{\mathrm{BB}}$ with pure ICRH or $\mathrm{LH}$}

Radial profiles of the BB contribution at different ranges of the edge safety factor and heating power are shown in Figures $10(\mathrm{ICRH})$ and $11(\mathrm{LH})$. The $E_{\mathrm{BB}}$ profiles in Ohmic plasmas with the same range of $q_{\psi}$ are shown for comparison.

From Figure 10, it is clear that $E_{\mathrm{BB}}$ is generally considerably higher with ICRH than in Ohmic plasmas, approaching the Ohmic $E_{\mathrm{BB}}$ only far outside the $q=1$ surface. Towards the LFS, almost all of the energy is in the broadband component. At the HFS and inside the central basin, $E_{\mathrm{BB}}$ increases with increasing heating power. At fixed heating power, a slight rise of $E_{\mathrm{BB}}$ is also observed with increasing $q_{\psi}$. This rise corresponds to an increase of the fluctuation level due to the degradation of the confinement with increasing $q_{\psi}$ (i.e. decreasing $I_{p}$ ). This rise has not been observed for the Ohmic cases, because $I_{p}$ is used as a heating source in Ohmic plasmas and 


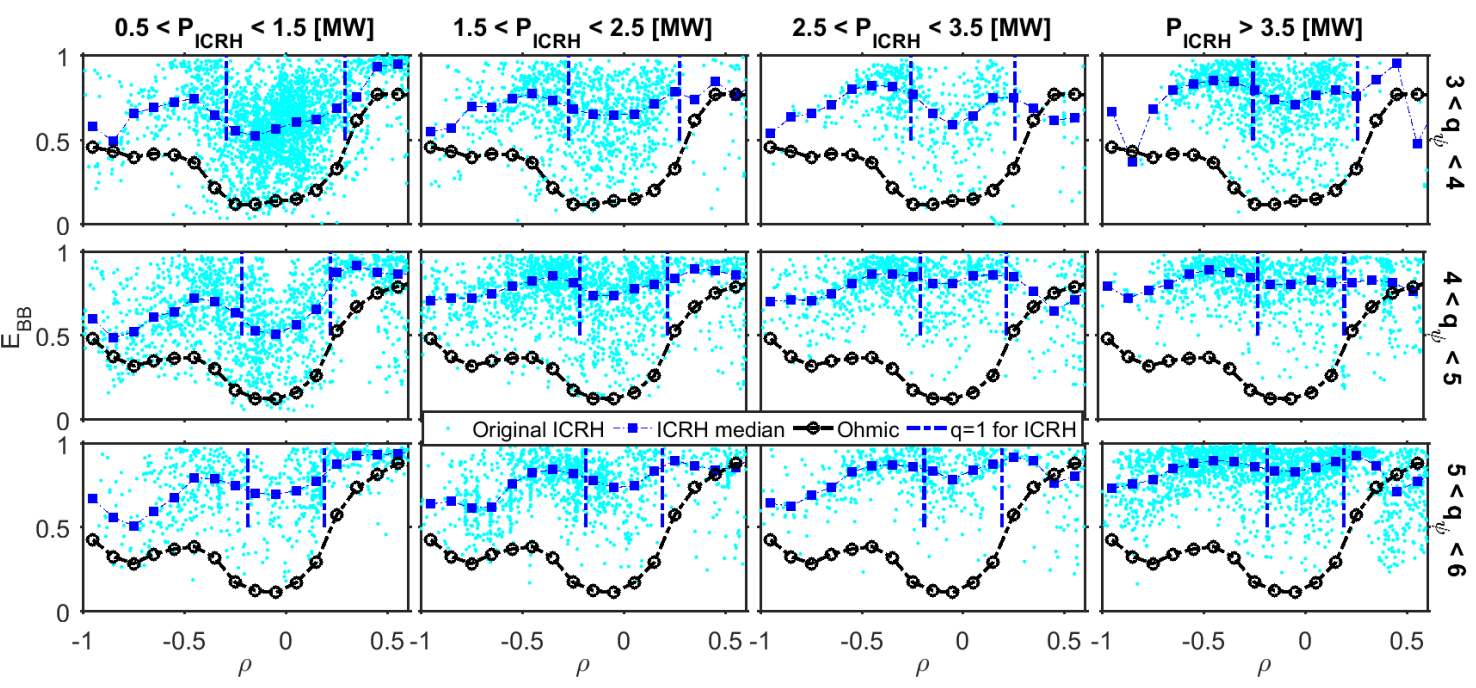

Figure 10. Radial profiles of $E_{\mathrm{BB}}$ in pure ICRH plasmas (blue) with various ranges of heating power and $q_{\psi}$. The $q=1$ surface for the ICRH plasmas is indicated by the vertical dashed lines in each panel. $E_{\mathrm{BB}}$ profiles in Ohmic plasmas (black) are shown for comparison.

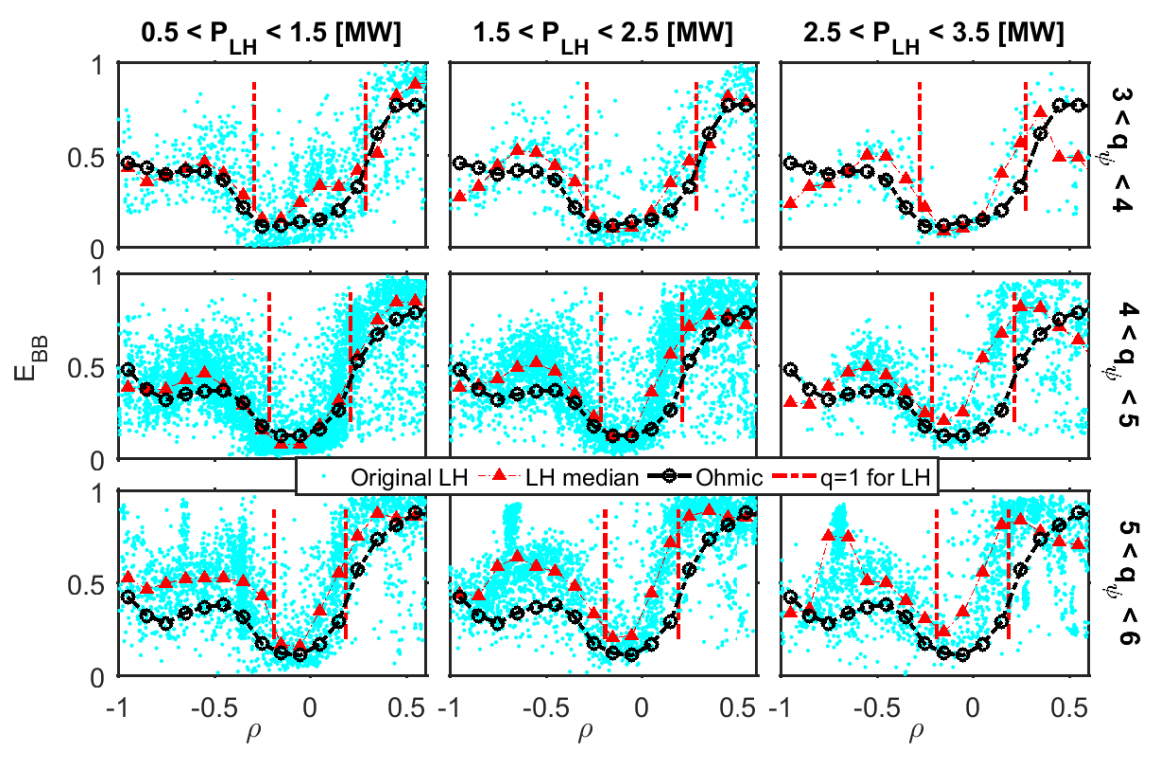

Figure 11. Similar to Figure 10, but for LH-heated plasmas (limited to $P_{\mathrm{LH}}=3.5 \mathrm{MW}$ ).

thus the situation is more complicated. Note that at the HFS near the edge, $E_{\mathrm{BB}}$ is clearly lower than in the core region. The higher $E_{\mathrm{BB}}$ in the core might be caused by the fast particles, as the ICRH power deposition occurs mainly in the core at Tore Supra. Specifically, at the lowest ICRH heating powers $\left(0.5 \mathrm{MW}<P_{\mathrm{ICRH}}<1.5 \mathrm{MW}\right)$, there is a clear $E_{\mathrm{BB}}$ basin in the core, but it becomes shallower with increasing $P_{\mathrm{ICRH}}$, and almost disappears when $P_{\text {ICRH }}>2.5 \mathrm{MW}$. This trend is observed for all $q_{\psi}$ ranges.

On the other hand, for plasmas with LH auxiliary heating, $E_{\mathrm{BB}}$ levels are comparable with the Ohmic case throughout the plasma cross-section at the lowest $q_{\psi}$. At higher heating powers or larger $q_{\psi}$ ranges, the $E_{\mathrm{BB}}$ pro- files with $\mathrm{LH}$ heating somewhat rise above the Ohmic case, but the difference remains relatively small. Accordingly, the basin remains clearly visible even at the highest LH heating power $\left(P_{\mathrm{LH}}>3 \mathrm{MW}\right)$.

Similar to the Ohmic case discussed in Section II, the above observations of $E_{\mathrm{BB}}$ profiles with ICRH or LH recall previous studies of density fluctuations. ${ }^{40}$ Most noticeably, in the central region and at the HFS, there are significant differences between the $E_{\mathrm{BB}}$ profiles in plasmas with ICRH vs. LH. In particular, in LH-heated plasmas, $E_{\mathrm{BB}}$ is limited, below $<0.5$, in the HFS and central region, especially in the latter $\left(0.1<E_{\mathrm{BB}}<0.2\right)$. In contrast, with ICRH, $E_{\mathrm{BB}}$ goes well above 0.5 for all radii, reaching levels of $0.7-0.8$ in the central region and at the 
HFS, and even higher at the LFS.

The distribution of the global energy confinement time $\left(\tau_{E}\right)$ with both heating methods (within the same range of heating power and edge safety factor) has been investigated systematically. The results show that $\tau_{E}$ remains almost the same for the two heating schemes. This indicates that the remarkable difference of $E_{\mathrm{BB}}$ between ICRH and LH (factor 5-6) cannot be explained by a degradation of confinement caused by enhanced turbulence, hence another mechanism must be at work.

\section{B. Evolution with collisionality}

To better understand the different levels of $E_{\mathrm{BB}}$ in Lmode with ICRH or LH heating, the evolution of $E_{\mathrm{BB}}$ with effective collisionality has been analyzed as well. Calculations of $\nu_{\text {eff }}$ are more complicated in L-mode, because measurements of $Z_{\text {eff }}$ from bremsstrahlung spectroscopy can be less reliable in Tore Supra LH discharges, reaching very large values $(\gtrsim 6)$. It was previously reported that hot spots on the inner wall, supra-thermal electrons and influence of reflections may cause overestimation of $Z_{\text {eff }}$ in Tore Supra LH plasmas. ${ }^{41}$ Therefore, a robust multi-machine scaling law ${ }^{42}$ has been used to obtain $Z_{\text {eff }}$ in LH plasmas:

$$
Z_{\mathrm{eff}}=1+7 \frac{P_{\mathrm{rad}}}{S n_{l}^{2}}
$$

Here, $P_{\text {rad }}$ is the total radiated power in MW, $S=4 \pi^{2} R a$ the plasma surface area in $\mathrm{m}^{2}$ and $n_{\mathrm{l}}$ the line-averaged density in $10^{-20} \mathrm{~m}^{-3}$, all available in the database. The distributions of $Z_{\text {eff }}$ values in ICRH from bremsstrahlung measurement on the one hand, and the scaling law on the other hand, are relatively similar. Nevertheless, the scaling law has also been used in ICRH to maintain consistency with the LH case.

\section{Dependence of $E_{\mathrm{BB}}$ on $\nu_{\mathrm{eff}}$}

Figure 12 shows the evolution of $E_{\mathrm{BB}}$ with $\nu_{\text {eff }}$ at different $\rho$. The value of $\nu_{\text {eff }}$ is systematically lower here than in Ohmic plasmas, due to the higher $T_{\mathrm{e}}$ with additional heating. Inside the central basin (Figure 12 (b)), the LH plasmas dominate in the low $\nu_{\text {eff }}$ ranges. In this region, most of $E_{\mathrm{BB}}$ in LH plasmas remains at a low level $(\sim 0.2)$ for $\nu_{\text {eff }}<0.3$, when Ohmic plasmas are in LOC regime (Figure 7 (b)). The ICRH plasmas dominate in the higher $\nu_{\text {eff }}$ ranges and most of $E_{\mathrm{BB}}$ in ICRH is above 0.5 with strong dispersion. Regardless of the heating method, the general increasing trend of $E_{\mathrm{BB}}$ with $\nu_{\mathrm{eff}}$ observed in Ohmic plasmas is recovered, not only inside but also outside the basin. Furthermore, at the LFS (Figure 12 (c)), $E_{\mathrm{BB}}$ in LH, and probably also ICRH, is close to saturation at large $\nu_{\text {eff }}$, while at the HFS (Figure 12 (a)) saturation of $E_{\mathrm{BB}}$ only occurs with ICRH. It should

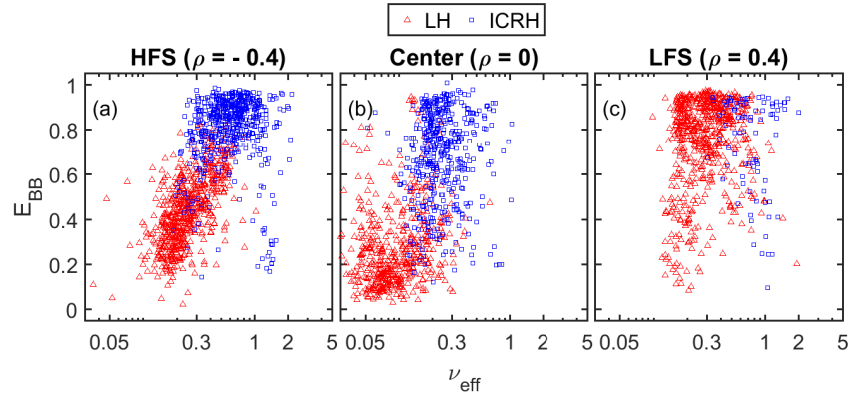

Figure 12. Evolution of the broadband contribution $\left(E_{\mathrm{BB}}\right)$ with effective collisionality $\left(\nu_{\text {eff }}\right)$ in L-mode with pure ICRH or LH at different radial positions. Only plasmas with moderate heating power $\left(1.5 \mathrm{MW}<P_{\text {heat }}<2.5 \mathrm{MW}\right)$ are shown.

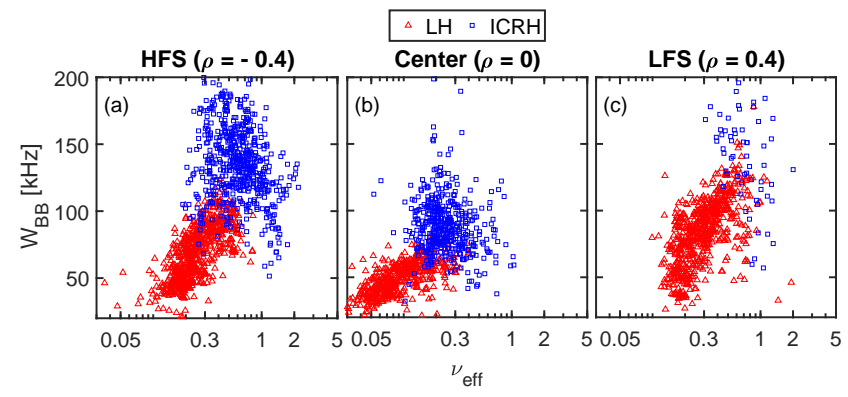

Figure 13. Same as Figure 12 for the evolution of the broadband width $W_{\mathrm{BB}}$.

be noted that relatively few plasmas are available in the database with ICRH at the LFS (Figure 12 (c)), somewhat degrading the reliability of the results under these conditions. This is due to a lack of valid spectra caused by a strong Doppler effect, which often affects the spectra towards the LFS.

\section{Dependence of $W_{\mathrm{BB}}$ on $\nu_{\mathrm{eff}}$}

We next study the evolution of the BB width $\left(W_{\mathrm{BB}}\right)$ with effective collisionality $\left(\nu_{\text {eff }}\right)$, in the presence of ICRH or LH. Figure 13 shows the dependence of $W_{\mathrm{BB}}$ on $\nu_{\mathrm{eff}}$ at different $\rho$. Generally, an increasing trend of $W_{\mathrm{BB}}$ with $\nu_{\text {eff }}$ can be identified. Specifically, for the LH plasmas, $W_{\mathrm{BB}}$ increases with $\nu_{\text {eff }}$ almost linearly when $\nu_{\text {eff }}$ is in the logarithmic scale. The BB width at the HFS and LFS follow a similar increasing trend, at a rate that is clearly higher than in the center. However, the BB width in ICRH plasmas has much stronger dispersion, although possibly a generally decreasing trend with collisionality can be discerned.

\section{Density peaking}

Figure 14 shows the evolution of the density peaking with respect to the effective collisionality. The den- 


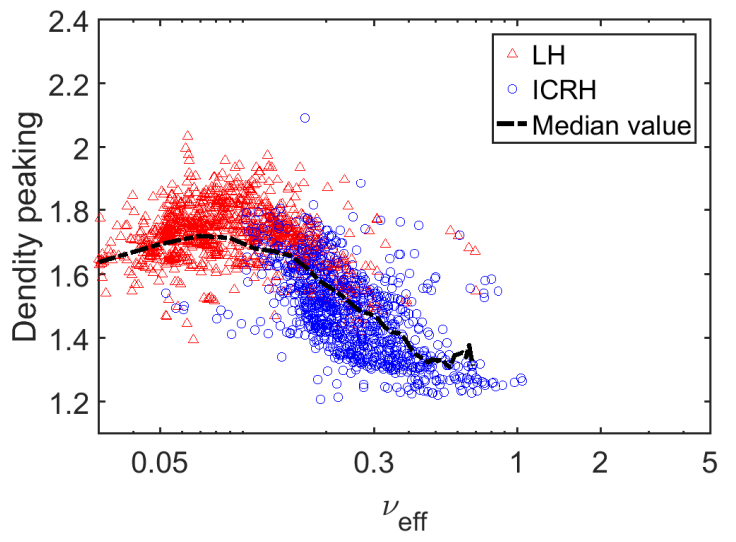

Figure 14. Evolution of the density peaking factor with respect to the effective collisionality with injected power

sity peaking increases slowly with $\nu_{\text {eff }}$ at lower $\nu_{\text {eff }}$, corresponding almost exclusively to LH-heated discharges, reaching a maximum value at about $\nu_{\text {eff }} \sim 0.1$. At higher $\nu_{\text {eff }}(>0.1)$, the density peaking decreases rapidly with $\nu_{\text {eff }}$, corresponding mostly with ICRH discharges. How-

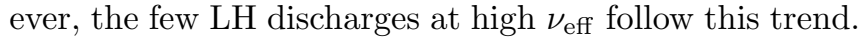
The evolution of the density peaking with $\nu_{\text {eff }}$ is thus very similar to the trends observed in the Ohmic case (Figure 6 ), expect that the slope at low $\nu_{\text {eff }}$ is much flatter in $\mathrm{LH}$ discharges than in the LOC regime.

\section{Interpretation}

Given the similarity of the observed trends of $E_{\mathrm{BB}}$ in terms of $\nu_{\text {eff }}$ with ICRH or LH, compared to the Ohmic case, one possible explanation is again offered by the stabilization of the TEM instabilities with increasing collisionality. At high collisionality, this would result in discharges where the turbulence would be driven mostly or exclusively by ITG instabilities. The decrease of the density peaking (Figure 14) with $\nu_{\text {eff }}$ above 0.1 supports this interpretation. Below this threshold, the density peaking increases with $\nu_{\text {eff }}$, but the slope is weaker than in Ohmic cases (Figure 6). This might be explained by the current driven by LH waves that reduce thus the toroidal electric field $E_{\phi}$. Another explanation could be that the turbulence is due to a mix of TEM and ITG instabilities at low $\nu_{\text {eff }}$ below the threshold. The decrease of the density peaking with $\nu_{\text {eff }}$ for ITG turbulence would reduce the slope at low $\nu_{\text {eff }}$ as observed in the LOC regime (Figure $6)$.

As for the $\mathrm{BB}$ width $\left(W_{\mathrm{BB}}\right)$, attempts have been made in the past to connect the width and shape of frequency spectra to the underlying instability, both from the theoretical and experimental viewpoints. ${ }^{43-45}$. With the extensive spectrum database, we have identified an increasing trend of the BB width with collisionality in L-mode plasmas with ICRH or LH heating, although no such gen- eral trends has been observed in the Ohmic case. The $\mathrm{BB}$ width could be linked with both turbulence properties and toroidal intrinsic rotation. In terms of the latter mechanism, one might expect a decrease of $W_{\mathrm{BB}}$ with increasing collisionality, when the main location where the power is deposited moves outward. The fact that, on the contrary, an increasing trend is observed for the LH plasmas in this database study (Figure 13), could indicate an unknown connection between the BB width and the underlying instabilities. The net effect could be the result of a competition between multiple mechanisms. Therefore, the link between the spectral width of the fluctuation measurements and the turbulence properties or instabilities remains an open question. Note that also the broadband shape could be an important factor in a systematic study, due to its connection with the BB width. Although only weak trends have been observed for the BB shape for both Ohmic and L-mode plasmas (not presented in this paper), other or additional plasma parameters might be used to simultaneously explain the $\mathrm{BB}$ width and shape in future work.

To put the proposed interpretation on firmer ground, one would need to identify a number of discharges in the database that are sufficiently diagnosed to enable transport analysis with a view to validated profiles of density, temperature, current and impurity concentrations. Then, quasi-linear gyrokinetic simulations with the QuaLiKiz code ${ }^{46}$ or with gyrokinetic codes such as GENE could be performed to compute the growth rates of the different instabilities and deduce the dominant ones. In a next step, ones would perform nonlinear gyrokinetic simulations to obtain density fluctuation maps. Full-wave reflectometry simulations can then be run to obtain frequency fluctuation spectra that could be compared to the experimental measurements.

\section{v. CONCLUSIONS AND DISCUSSION}

Using a new parametrization method ${ }^{21}$, we have built a large database with more than 300,000 reflectometry frequency spectra. A number of dependencies of the spectra have been studied in Ohmic and L-mode plasmas. Globally, the $\mathrm{BB}$ component contributes more to the reflectometry spectra in the SOC regime than in LOC regime. In pure ICRH discharges, the BB component contains almost all energy in the spectrum, whereas its contribution is much weaker in pure LH discharges. The LF component is also wider in SOC and ICRH discharges, compared to, respectively, LOC and LH discharges. Moreover, general increasing trends of the $\mathrm{BB}$ contribution $\left(E_{\mathrm{BB}}\right)$ with effective collisionality $\left(\nu_{\text {eff }}\right)$ have been identified in both Ohmic plasmas and L-mode plasmas with ICRH and LH heating. Trends of the BB width $\left(W_{\mathrm{BB}}\right)$ in terms of $\nu_{\text {eff }}$ are opposite for plasmas with LH heating (increasing) vs. ICRH heating (decreasing). Changes in the density fluctuation spectra with a change of the dominant instabilities or reduction of the TEM contri- 
bution with increasing $\nu_{\text {eff }}$, could explain these observations. Trends in the density peaking in terms of $\nu_{\text {eff }}$ support this interpretation.

Apart from identification of global trends, another feature of a database study lies in its ability to detect specific clusters or outliers. An example in this study is the obvious cluster in Figure 7 (c) with low $E_{\mathrm{BB}}$, as well as the group of outliers in Figure 6 with $\nu_{\text {eff }} \sim 1$. The clusters usually correspond to a limited number of discharges with possibly exceptional experimental conditions, but might also point at interesting physics. A database study allows identifying such clusters in a straightforward way.

The trends that have been observed through the present database study in combination with an efficient spectrum parametrization, must be confirmed through simulations and dedicated experiments, taking into account that some circumstances have changed with the upgrade from Tore Supra to the WEST tokamak. Furthermore, there are many ways in which the present study could be improved. The strong dispersion of the data suggest that additional plasma parameters will have to be employed to better order the data and characterize trends of the fluctuation spectra. On the other hand, while the individual parameters used to characterize each spectrum may be interpreted from the physical point of view, each of them only quantifies a certain aspect of the spectrum. The parametrization method could be augmented with modern techniques from data science, enabling a more integrated quantification of shapes and distributions. ${ }^{47}$ Combined with advanced regression analysis, this could contribute to systematic studies of the characteristics of micro-instabilities in terms of varying plasma conditions. Equipped with a similarity measure between frequency spectra, classification techniques could help discriminating between turbulent regimes based on the shape of their spectra.

The observed trends confirm the relevance of the database approach, which is complimentary to the more traditional shot-to-shot analysis. Ongoing tremendous developments in data storage, data processing capabilities, as well as analysis and modeling techniques are rapidly impacting a broad variety of scientific domains. Such developments are expected to lead to new discoveries and a deeper understanding in fusion science as well.

\section{ACKNOWLEDGMENTS}

The authors wish to thank P. Devynck, J. F. Artaud and C. Bourdelle for the instructive discussions. This work has been carried out within the framework of the French Federation for Magnetic Fusion Studies (FR-FCM) and of the EUROfusion Consortium, and has received funding from the Euratom research and training programme 2014-2018 and 2019-2020 under grant agreement No 633053. The views and opinions expressed herein do not necessarily reflect those of the European Commission. This work has been carried out within the framework of the Erasmus Mundus International Doctoral College in Fusion Science and Engineering (FUSION-DC).

\section{REFERENCES}

${ }^{1}$ M. Greenwald, D. Gwinn, S. Milora, J. Parker, R. Parker, S. Wolfe, M. Besen, F. Camacho, S. Fairfax, C. Fiore, et al., Phys. Rev. Lett. 53, 352-355 (1984).

${ }^{2}$ Y. R. Martin, T. Takizuka, and the ITPA CDBM H-mode Threshold Database Working Group, Journal of Physics: Conference Series 123, 012033 (2008).

${ }^{3}$ P. Yushmanov, T. Takizuka, K. Riedel, O. Kardaun, J. Cordey, S. Kaye, and D. Post, Nucl. Fusion 30, 1999 (1990).

${ }^{4}$ G. Verdoolaege and J.-M. Noterdaeme, Nucl. Fusion 55, 113019 (2015).

${ }^{5}$ A. E. White, L. Schmitz, G. R. McKee, C. Holland, W. A. Peebles, T. A. Carter, M. W. Shafer, M. E. Austin, K. H. Burrell, J. Candy, et al., Phys. Plasmas 15, 056116 (2008).

${ }^{6}$ C. Sung, A. E. White, D. R. Mikkelsen, M. Greenwald, C. Holland, N. T. Howard, R. Churchill, and C. Theiler, Phys. Plasmas 23, 042303 (2016).

${ }^{7}$ C. Holland, Phys. Plasmas 23, 060901 (2016).

${ }^{8}$ A. J. Creely, N. T. Howard, P. Rodriguez-Fernandez, N. Cao, A. E. Hubbard, J. W. Hughes, J. E. Rice, A. E. White, J. Candy, G. M. Staebler, et al., Phys. Plasmass 24, 056104 (2017).

${ }^{9}$ C. C. Petty, T. C. Luce, K. H. Burrell, S. C. Chiu, J. S. deGrassie, C. B. Forest, P. Gohil, C. M. Greenfield, R. J. Groebner, R. W. Harvey, et al., Phys. Plasmas 2, 2342-2348 (1995).

${ }^{10}$ J. Christiansen, P. Stubberfield, J. Cordey, C. Gormezano, C. Gowers, J. O'Rourke, D. Stork, A. Taroni, and C. Challis, Nucl. Fusion 33, 863 (1993).

${ }^{11}$ M. Vlad and F. Spineanu, Plasma Phys. Control. Fusion 47, 281 (2005).

12 E. Mazzucato and R. Nazikian, Plasma Phys. Control. Fusion 33, 261 (1991)

${ }^{13}$ R. Nazikian, G. J. Kramer, and E. Valeo, Phys. Plasmas 8, 1840 (2001).

${ }^{14}$ E. Gusakov, S. Heuraux, A. Popov, and M. Schubert, Plasma Phys. Control. Fusion 54, 045008 (2012).

${ }^{15}$ D. A. Shelukhin, S. V. Soldatov, V. A. Vershkov, and A. O. Urazbaev, Plasma Physics Reports 32, 707-717 (2006).

${ }^{16}$ N. V. Kosolapova, E. Z. Gusakov, and S. Heuraux, Plasma Phys. Control. Fusion 54, 035008 (2012).

${ }^{17}$ V. A. Vershkov, D. A. Shelukhin, S. V. Soldatov, A. O. Urazbaev, S. A. Grashin, L. G. Eliseev, A. V. Melnikov, and the T-10 team, Nucl. Fusion 45, S203 (2005).

${ }^{18}$ V. A. Vershkov, A. Borschegovskiy, V. Chistyakov, M. Dremin, L. Eliseev, E. Gorbunov, S. Grashin, A. Khmara, A. Kislov, D. Kislov, et al., Nucl. Fusion 51, 094019 (2011).

${ }^{19}$ A. Krämer-Flecken, V. Dreval, S. Soldatov, A. Rogister, V. Vershkov, and the TEXTOR-team, Nucl. Fusion 44, 1143 (2004).

${ }^{20}$ A. Krämer-Flecken, S. Soldatov, Y. Xu, H. Arnichand, S. Hacquin, R. Sabot, and the TEXTOR team, New Journal of Physics 17, 073007 (2015).

${ }^{21}$ Y. Sun, R. Sabot, G. Hornung, S. Heuraux, S. Hacquin, and G. Verdoolaege, Rev. Sci. Instrum. 89, 073504 (2018).

${ }^{22}$ R. Sabot, A. Sirinelli, J.-M. Chareau, and J.-C. Giaccalone, Nucl. Fusion 46, S685 (2006).

${ }^{23}$ P. Hennequin, C. Honoré, A. Quéméneur, A. Truc, F. Gervais, C. Fenzi, and R. Sabot, in 26th EPS Conf. on Contr. Fusion and Plasma, Vol. 23J (European Physical Society, Petit-Lancy, 1999) pp. $977-980$.

${ }^{24}$ H. Arnichand, R. Sabot, S. Hacquin, A. Krämer-Flecken, X. Garbet, J. Citrin, C. Bourdelle, G. Hornung, J. Bernardo, C. Bottereau, et al., Nucl. Fusion 54, 123017 (2014).

${ }^{25}$ R. Sabot, F. Clairet, G. D. Conway, L. Cupido, X. Garbet, 
G. Falchetto, T. Gerbaud, S. Hacquin, P. Hennequin, S. Heuraux, et al., Plasma Phys. Control. Fusion 48, B421 (2006).

${ }^{26}$ G. Hornung, F. Clairet, G. L. Falchetto, R. Sabot, H. Arnichand, and L. Vermare, Plasma Phys. Control. Fusion 55, 125013 (2013).

${ }^{27}$ V. Arunasalam, N. Bretz, P. Efthimion, R. Goldston, B. Grek, D. Johnson, M. Murakami, K. McGuire, D. Rasmussen, F. Stauffer, et al., Nucl. Fusion 30, 2111 (1990).

${ }^{28}$ Y. Shimomura, N. Suzuki, M. Sugihara, K. Odajima, and T. Tsunematsu, JAERI Report 87-080 (1987).

${ }^{29}$ C. Gil, C. D. Michelis, D. Elbeze, C. Fenzi, J. P. Gunn, F. Imbeaux, P. Lotte, D. Mazon, O. Meyer, M. Missirlian, et al., Fusion Science and Technology 56, 1219-1252 (2009).

${ }^{30}$ C. Angioni, A. G. Peeters, F. Ryter, F. Jenko, G. D. Conway, T. Dannert, H. U. Fahrbach, M. Reich, W. Suttrop, and L. Fattorini, Phys. Plasmas 12, 040701 (2005).

${ }^{31}$ I. Erofeev, E. Fable, C. Angioni, R. McDermott, and T. A. U. Team, Nucl. Fusion 57, 126067 (2017).

${ }^{32}$ J. E. Rice, M. J. Greenwald, Y. A. Podpaly, M. L. Reinke, P. H. Diamond, J. W. Hughes, N. T. Howard, Y. Ma, I. Cziegler, B. P. Duval, et al., Phys. Plasmas 19, 056106 (2012).

${ }^{33}$ A. Lebschy, R. McDermott, C. Angioni, B. Geiger, D. Prisiazhniuk, M. Cavedon, G. Conway, R. Dux, M. Dunne, A. Kappatou, T. Pütterich, U. Stroth, E. Viezzer, and the ASDEX Upgrade Team, Nucl. Fusion 58, 026013 (2018).

${ }^{34}$ C. Angioni, A. G. Peeters, G. V. Pereverzev, F. Ryter, and G. Tardini, Phys. Plasmas 10, 3225-3239 (2003).

${ }^{35}$ X. Garbet, P. Mantica, C. Angioni, E. Asp, Y. Baranov, C. Bourdelle, R. Budny, F. Crisanti, G. Cordey, L. Garzotti, et al., Plasma Phys. Control. Fusion 46, B557 (2004).

${ }^{36}$ G. D. Conway, C. Angioni, R. Dux, F. Ryter, A. Peeters,
J. Schirmer, C. Troester, C. R. Group, and the ASDEX Upgrade team, Nucl. Fusion 46, S799 (2006).

${ }^{37}$ S. Hacquin, J. Citrin, H. Arnichand, R. Sabot, C. Bourdelle, X. Garbet, and A. Krämer-Flecken, Phys. Plasmas 23, 092303 (2016).

${ }^{38}$ J. Citrin, H. Arnichand, J. Bernardo, C. Bourdelle, X. Garbet, F. Jenko, S. Hacquin, M. J. Pueschel, and R. Sabot, Plasma Phys. Control. Fusion 59, 064010 (2017).

${ }^{39}$ H. Arnichand, J. Citrin, S. Hacquin, R. Sabot, A. KrämerFlecken, X. Garbet, C. Bourdelle, C. Bottereau, F. Clairet, J. C. Giacalone, et al., Plasma Phys. Control. Fusion 58, 014037 (2016)

${ }^{40}$ R. Guirlet, A. Sirinelli, T. P. b, R. Sabot, J. Artaud, C. Bourdelle, X. Garbet, P. Hennequin, G. Hoang, F. Imbeaux, et al., Nucl. Fusion 50, 095009 (2010).

${ }^{41}$ B. Schunke, G. T. A. Huysmans, and P. R. Thomas, Rev. Sci. Instrum. 76, 083501 (2005).

${ }^{42}$ G. Matthews, S. Allen, N. Asakura, J. Goetz, H. Guo, A. Kallenbach, B. Lipschultz, K. McCormick, M. Stamp, U. Samm, et al., Journal of Nuclear Materials 241-243, 450 - 455 (1997).

${ }^{43}$ F. Romanelli, Physics of Fluids B: Plasma Physics 1, 1018-1025 (1989).

${ }^{44}$ N. Mattor and P. W. Terry, Physics of Fluids B: Plasma Physics 4, 1126-1138 (1992).

${ }^{45}$ C. Watts, R. F. Gandy, G. Cima, R. V. Bravenec, D. W. Ross, A. J. Wootton, A. Ouroua, J. W. Heard, T. P. Crowley, P. M. Schoch, et al., Phys. Plasmas 3, 2013-2021 (1996).

${ }^{46}$ C. Bourdelle, X. Garbet, F. Imbeaux, A. Casati, N. Dubuit, R. Guirlet, and T. Parisot, Phys. of Plasmas 14, 112501 (2007).

${ }^{47}$ A. Shabbir, G. Hornung, G. Verdoolaege, and JET Contributors, Rev. Sci. Instrum. 87, 11D404 (2016). 